OPEN ACCESS

Edited by:

Hisashi Nishigawa,

Utsunomiya University, Japan

Reviewed by: Katarzyna Otulak-Koziet, Warsaw University of Life

Sciences - SGGW, Poland

Muhammad Ali,

Zhejiang University, China

*Correspondence:

Yong Liu

haoasliu@163.com

Specialty section:

This article was submitted to

Virology,

a section of the journal

Frontiers in Microbiology

Received: 15 March 2021

Accepted: 28 May 2021

Published: 13 September 2021

Citation

Wang L, Tian P, Yang $X$, Zhou $X$,

Zhang S, Li C, Yang $X$ and Liu Y

(2021) Key Amino Acids for Pepper

Vein Yellows Virus PO Protein

Pathogenicity, Gene Silencing,

and Subcellular Localization.

Front. Microbiol. 12:680658.

doi: 10.3389/fmicb.2021.680658

\section{Key Amino Acids for Pepper Vein Yellows Virus PO Protein Pathogenicity, Gene Silencing, and Subcellular Localization}

\author{
Lishuang Wang 1,2, Peijie Tian', Xiuling Yang ${ }^{3}$, Xueping Zhou ${ }^{3}$, Songbai Zhang ${ }^{4}$, Chun Li², \\ Xuehui Yang ${ }^{2}$ and Yong Liu1,4*
}

${ }^{1}$ College of Plant Protection, Hunan Agricultural University, Changsha, China, ${ }^{2}$ Institute of Plant Protection, Guizhou Academy of Agricultural Sciences, Guiyang, China, ${ }^{3}$ State Key Laboratory for Biology of Plant Diseases and Insect Pests, Institute of Plant Protection, Chinese Academy of Agricultural Sciences (CAAS), Beijing, China, ${ }^{4}$ Institute of Plant Protection, Hunan Academy of Agricultural Sciences, Changsha, China

Pepper vein yellows virus (PeVY) is a newly recognized Polerovirus extracted from Chinese pepper. The symptoms of PeVYV-infested pepper plants comprise intervein yellow staining, leaf curl formation and other malformations, and leaf internodal shrinkage, but the roles of the viral proteins remain undetermined. The PO protein of the genus Polerovirus has established post-transcriptional gene silencing (PTGS) activity. This investigation focused on the PeVYV-encoded PO protein and assessed its potential virulence capacity, PTGS activity, and tendencies to localize in the nucleus. This study revealed that $\mathrm{PO}$ influenced the pathogenic properties of a specific heterologous potato virus $X$. In addition, PO proteins impaired local gene silencing, although they did not regulate generalized gene silencing within Nicotiana benthamiana 16c plants. Furthermore, PO proteins localized mainly in the nucleus, particularly in the nucleolus. PO deletion mutagenesis demonstrated that the F-box motif (56-72 amino acids, AAs) of PO was essential for symptom determination, inhibition of PTGS, and subcellular localization. Mutation analysis of the F-box motif of PO protein indicated that AA 57 of the PO protein was a pivotal site in symptom development and that AA 56 of the PO protein was indispensable for inhibiting PTGS and subcellular localization. The outcomes obtained here suggest that further studies should be conducted on the molecular mechanisms of amino acids of the F-box domain of PO protein in the interaction of PeVYV with plants.

Keywords: pepper vein yellows virus, Polerovirus, pathogenic factor, RNA silencing suppressor, subcellular localization

\section{INTRODUCTION}

Pepper (Capsicum spp.) is a major agricultural crop grown in China, but one of its main biotic threats is pepper vein yellow virus (PeVYV), which is a notorious pathogen due to its geographical distribution and its linkage to severe agricultural crop yield losses. PeVYV was initially identified in Japan in 1995 and is transmitted by the Aphis gossypii Glover aphid (Yonaha et al., 1995). The symptoms of PeVYV comprise intervein yellow staining, leaf curl formation and other malformations, leaf puckering, and internodal shrinking. Such symptom traits have also been identified in pepper, tomato, tobacco, black nightshade, and other 
solanaceous plants from a spectrum of regions within Africa, Asia, and Europe (Dombrovsky et al., 2010; Murakami et al., 2011; Knierim et al., 2013; Villanueva et al., 2013; Alfaro et al., 2014; Alabi et al., 2015; Tan et al., 2015; Zhang et al., 2015; Liu et al., 2016; Afouda et al., 2017; Wang et al., 2017).

Pepper vein yellow virus belongs to the family Luteoviridae of the genus Polerovirus, whose genomes consist of single linear, positive-sense, single-stranded RNA containing 6,244 nucleotides (nt), including six open reading frames (ORFs; ORF0 to ORF5) (Liu et al., 2016). ORF0 expresses an RNA-silencing suppressor protein, P0, whereas ORF1 and ORF2 express viral replication proteins such as viral RNA polymerase. The main coat protein (CP) is expressed from ORF3 (3' section). The ORF4 region, which is present within the $\mathrm{CP}$ gene, although on an alternative reading frame, leads to the expression of the viral movement protein. ORF5 can be expressed through casual CP termination codon suppression and also encodes the read-through domain, which is necessary for effective aphid transmission (Ali et al., 2018; Agrofoglio et al., 2019; Avelar et al., 2020).

Throughout the past few years, P0 proteins derived from multiple poleroviruses have been demonstrated to act as RNA silencing suppressors (RSSs) (Almasi et al., 2015; Cascardo et al., 2015; Chen et al., 2016; Wang et al., 2018; Agrofoglio et al., 2019; Li et al., 2019). Multiple investigations have assessed the necessity for an F-box-like motif or P0-S phase kinase-associated protein 1 (SKP1) involvement in RNA silencing inhibition (Almasi et al., 2015). The polerovirus-F-box P0 protein can induce AGONAUTE 1 (AGO1) protein disintegration as a viral countermeasure. The implementation of silencing inhibitors is typically synonymous with virulence traits. Mutations of the F-box-like motif mostly occur in tandem with the loss of HR elicitations, and both of these effects lead to a reduction in Turnip yellow virus (TuYV) P0 viral suppressors of RNA silencing/RNA interference (VSR) activities (Wang et al., 2015). The consensus minimal F-box-like motif (LPXX(L/I) X10-13P) in $\mathrm{P} 0$ proteins has been postulated to be essential for RNA silencing inhibition because its mutation affects P0-based RNA silencing inhibition properties (Pazhouhandeh et al., 2006). Nevertheless, the amino acid(s) involving the function(s) of the P0 protein still remains unclear.

Through P0 protein amino acid sequence analyses using samples obtained in Guizhou Province in China, its unique F-box motif could be identified (56 LPFLLSSQCPFLSGNTP72). The purpose of this study was to elucidate how the action of P0 could also provide a strategic angle of knowledge regarding the virulence roles of this protein as well as - ultimately - Polerovirus pathogenesis. This investigation assessed virulence variables and RNA silencing inhibitors, all of which were derived from the PeVYV P0 protein.

\section{MATERIALS AND METHODS}

\section{Plasmid Design and Development}

The P0 ORF was amplified from complementary DNA (cDNA) (Guizhou variant). The polymerase chain reaction (PCR) products were individually cloned into the pGEM-T Easy vector ${ }^{\circledR}$ (Transgen $^{\mathrm{TM}}$, Beijing, China) to generate pGEM-T-P0, which served as a substrate for specific enzymes to allow cloning. The mutant $\mathrm{P} 0^{d m 56-72 a a}$, in which the nucleotide sequences of 56-72 amino acid residues of the P0 protein were deleted, was constructed using the overlap PCR method (Bryksin and Matsumura, 2018). The mutants $\mathrm{P}^{\triangle} 56,57 a a, \mathrm{P}^{\triangle}{ }^{\triangle} 56 a$, and $\mathrm{P} 0^{\triangle 57 a}$, in which the amino acid(s) were replaced by arginine (A), were manufactured through the Fast Mutagenesis System ${ }^{\circledR}$ (Transgen $^{\mathrm{TM}}$, Beijing, China) using unique primer sets for on/around mutation sites (Table 1). To test for pathogenicity, the P0 ORF, together with mutated variants, was cloned within a PVX-containing pGR106 vector (mid-sequence inside ClaI and SalI restriction site boundaries) to yield PVX-P0.

The end products consisting of recombinant PVX constructs were separately transformed through electroporation within Agrobacterium tumefaciens strain GV3101.

For post-transcriptional gene silencing (PTGS) inhibition investigations, full-length ORFs of $\mathrm{P} 0$ or $\mathrm{P} 0^{d m 56-72 a a}, \mathrm{P}^{\triangle 56,57 a a}$, $\mathrm{P}^{\triangle} 56 a$, and $\mathrm{P}^{\triangle} 57 a$ were subcloned into the pCHF3 vector ${ }^{[30]}$ between the BamHI and SalI sites for the creation of pCHF3-

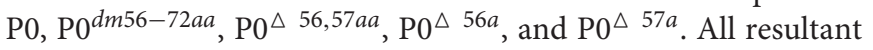
constructs were separately transformed into the A. tumefaciens strain C58C1 through electroporation.

For subcellular localization analyses, the full-length segment

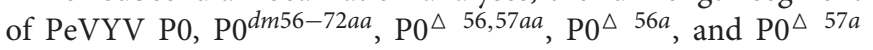
was incorporated within the BamHI and SalI sites of the pCHF3$\mathrm{N}-\mathrm{eGFP}$ vector ${ }^{[31]}$ to prepare $35 \mathrm{~S}-\mathrm{GFP}-\mathrm{P} 0, \mathrm{P} 0^{d m 56-72 a a}, \mathrm{P}^{\triangle}$ $56,57 a a, \mathrm{P}^{\triangle} 56 a$, and $\mathrm{P} 0^{\triangle} 57 a$, which contain $\mathrm{P} 0, \mathrm{P} 0$ dm56-72aa, $\mathrm{P}^{\triangle} 56,57 a a, \mathrm{P}^{\triangle} 56 a$, and $\mathrm{P}^{\triangle} 57 a$ viral $\mathrm{N}$-terminal fusion protein attached to an enhanced green fluorescent protein (eGFP), respectively. The resultant plasmid was transformed within A. tumefaciens strain C58C1 through electroporation measures.

\section{Agroinfiltration}

After incubation in Luria-Bertani broth with complementary antibiotics at $28^{\circ} \mathrm{C}$ overnight, all preparations were subjected to centrifugation/resuspension using infiltration buffer consisting of 10-mM 2-(N-morpholino)ethanesulfonic acid pH 5.7, 10-mM magnesium chloride, and 150-mM acetosyringone, after reaching an optical density at a wavelength of $600 \mathrm{~nm}$ of $0.5-1.0$. After incubation at $25^{\circ} \mathrm{C}$ for $3 \mathrm{~h}$, all suspensions were allowed to infiltrate within 4-week-old Nicotiana benthamiana foliage. At 36-h post-infiltration, these crops were consequently scrutinized for fluorescent properties by confocal laser scanning microscopy.

\section{Hydrogen Peroxide Detection}

This analysis was performed with $N$. benthamiana foliage using the 3,3'-diaminobenzidine (DAB)- hydrochloric acid collection methodology, according to previously reported protocols with minimal optimizations (Balzergue et al., 2017). In brief, the leaves were detached, treated with reagents, incubated overnight, bleached using $96 \%$ alcohol in boiling-hot water for $5 \mathrm{~min}$, and consequently subjected to fluorescence microscopy/photography. Such a technique was necessary for leaf discoloration and allowed consequent identification of hydrogen peroxide intensity distributions based on dark-brown sediments 
derived from interactions between $\mathrm{DAB}$ (reagent used in the study) and hydrogen peroxide $\left(\mathrm{H}_{2} \mathrm{O}_{2}\right)$.

\section{Post-transcriptional Gene Silencing Assay}

To analyze the efficiency of silencing inhibition, equivalent volumes of Agrobacterium cultures containing 35S-GFP and analyzed constructs were combined into one solution and then infiltrated within mature foliage of 4-week-old 16c (or wildtype) $N$. benthamiana plants. Concomitant 35S-GFP infiltration utilizing a construct expressing tomato bushy stunt virus P19 was used as a positive control, whereas an empty $\mathrm{pCHF} 3$ vector served as a negative control. A. tumefaciens cultures bearing 35S-GFP, together with $\mathrm{pCHF} 3-\mathrm{P} 0$ and its mutant variants, $\mathrm{P} 19$ construct, or the empty pCHF3 vector, were combined in equivalent fractions and allowed to infiltrate $N$. benthamiana $16 \mathrm{c}$ foliage. GFP fluorescence within infiltrated/non-infiltrated foliage was closely supervised with the aid of a UV lamp (Black-Ray ${ }^{\circledR}$ Model B-100A, San Gabriel, CA, United States).

\section{RNA Extraction and Analysis}

Total RNA was collected through TRIzol $^{\circledR}$ according to the manufacturer's protocols (Invitrogen ${ }^{\mathrm{TM}}$, Carlsbad, CA, United States). All heavily affected foliage of $N$. benthamiana crops treated with PVX or recombinant PVX constructs was collected and prepared for PeVYV P0 expression through quantitative reverse transcription PCR (RT-qPCR) measures. A $1-\mu \mathrm{g}$ mass of total RNA was reverse transcribed into cDNA using TransScript ${ }^{\circledR}$ II One-Step gDNA Removal and cDNA Synthesis SuperMix (Transgen ${ }^{\mathrm{TM}}$, Beijing, China). Individual PeVYV P0/mutant expression levels were analyzed using PCR using primer sets (listed in Table 1). $\rho$-Values were calculated using unpaired two-tailed Student's $t$-test (Li et al., 2019).

\section{Protein Extraction and Western Blotting}

Protein extractions (total soluble), sodium dodecyl sulfatepolyacrylamide gel electrophoresis, and Western blotting analyses were conducted according to previously described protocols (Agrofoglio et al., 2019). PVX recognition was conducted by extracting the proteinaceous content from heavily infected (with PVX or PVX recombinant constructs) $N$. benthamiana leaves. Anti-CP monoclonal antibody $(\mathrm{MAb})$ that targets PVX (developed at the Institute of Biotechnology, Zhejiang University, Hangzhou, China) was used (dilution factor $=1: 8,000)$. GFP determination was performed by extracting proteins from infiltrated foliage of $N$. benthamiana line $16 \mathrm{c}$.

All Western blotting assays were visualized using a secondary peroxidase-conjugated goat-based, anti-mouse antibody (Cell Signaling Technology ${ }^{\mathrm{TM}}$, Boston, MA, United States) together with a chemiluminescence detection system (Tianneng ${ }^{\mathrm{TM}}$, Shanghai, China).

\section{Fluorescence Analysis}

To determine the subcellular localizations of GFP fusion proteins, fluorescent imaging of $N$. benthamiana afflicted with transformed Agrobacterium was conducted through Zeiss ${ }^{\text {TM }}$ LSM $880^{\circledR}$ confocal laser scanning microscopy using presets for GFP (excitation at $488 \mathrm{~nm} / \mathrm{emission}$ at $500-550 \mathrm{~nm}$ ) and chloroplast autofluorescence (excitation at $561 \mathrm{~nm} /$ emission at 650-750 nm).

\section{RESULTS}

\section{PeVYV PO Is a Pathogenicity Determinant}

Following the research protocols described earlier, PVX-infused leaves developed mosaic symptoms in upward leaves visible at $10 \mathrm{dpi}$, and PVX-P0-infected plants developed symptoms of

TABLE 1 | Synthetic oligonucleotide primers.

Primers

Primers used for the construction of recombinant PVX vector or pCHF3-based binary vectors

PO-BamHI Clal-F

PO-Sall-R

Primers used to generate PO mutant

POdm56-72aa $-\mathrm{F}$

$P 0^{d m 56-72 a a}-R$

$\mathrm{PO}^{\Delta 56,57 a a_{-} \mathrm{F}}$

$\mathrm{PO}^{\Delta 56,57 a a_{-} \mathrm{R}}$

$P 0^{\Delta 56 a}-F$

$P 0^{\Delta 56 a}-R$

$P 0 \Delta 57 a-F$

$\mathrm{PO}^{\Delta 57 a}-\mathrm{R}$

Primers used for $\mathrm{qPCR}$

qPVX-F

qPVX-R

GAPDH-F

GAPDH-R
GGATCCATCGATATGAACTITGAATTGATCAACGGA

ACGCGTCGACTCACTGTAGTTCCTTCTGAATCTG

\section{CACCGGAACGGCAAGCGGGAACAG}

GAGAGCACAAATAGAGCGAAGAAAATGG

CTTCGCTCTATTTGTGCTCTCGCCGCTTCCTTCTCA

AGCGGCGAGAGCACAAATAGAGCGAAGAAAATGG

CTTCGCTCTATTTGTGCTCTCGCCCTITCCTTCTCA

AAGGGCGAGA GCACAAATAG AGCGAAGAAA ATGG

CTTCGCTCTATTTGTGCTCTCTCCGCTTCCTTCTC

AGCGGAGAGAGCACAAATAGAGCGAAGAAAATGG

CAGGGTCAACTACCTCAACTAC

GGCACGAGCTGTACTAAAGAA

GCAGTGAACGACCCATTTATCTC

AACCTTCTTGGCACCACCCT

Bold represents the endonuclease sites. 

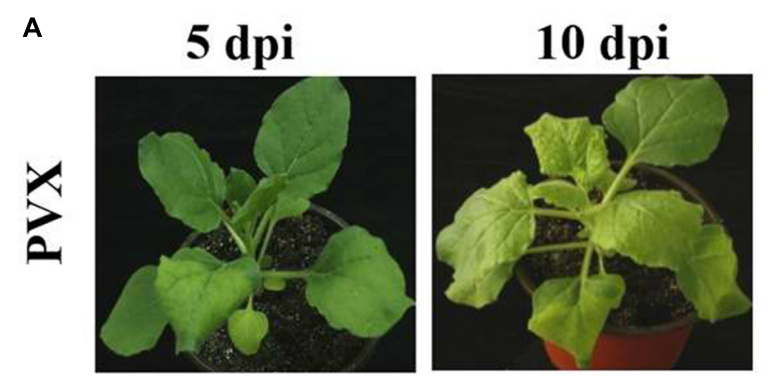

B
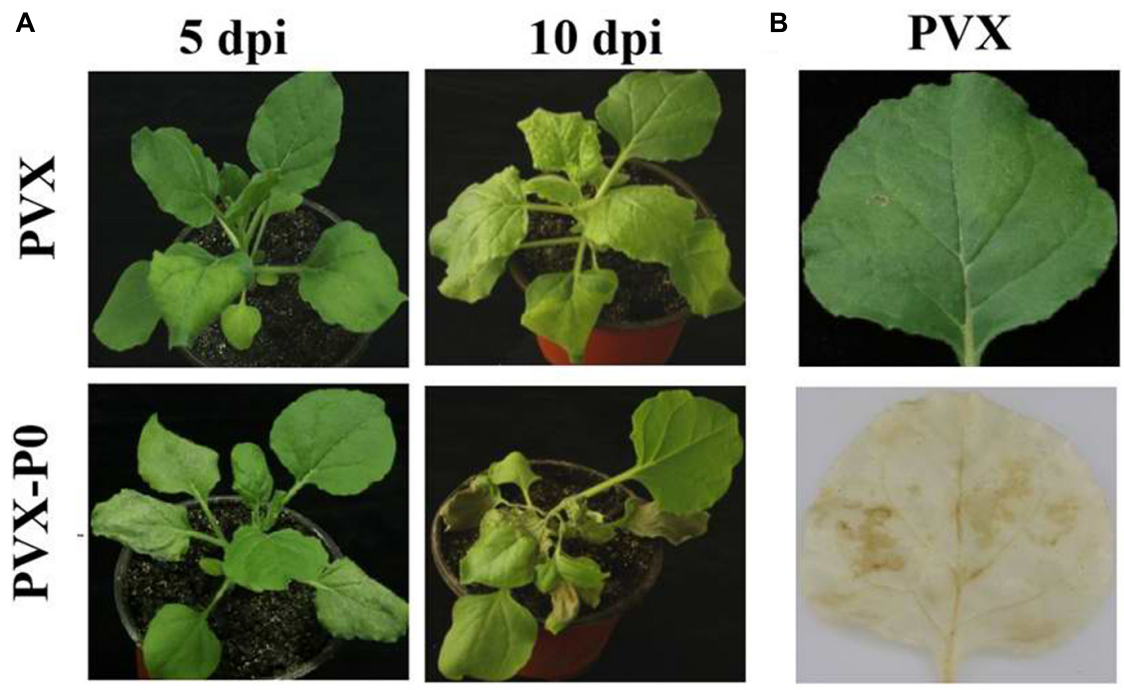

PVX-P0
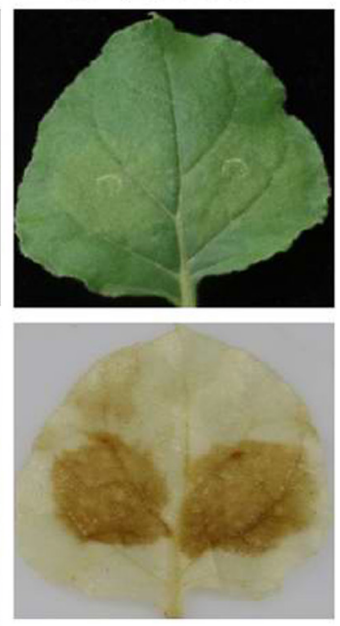

C

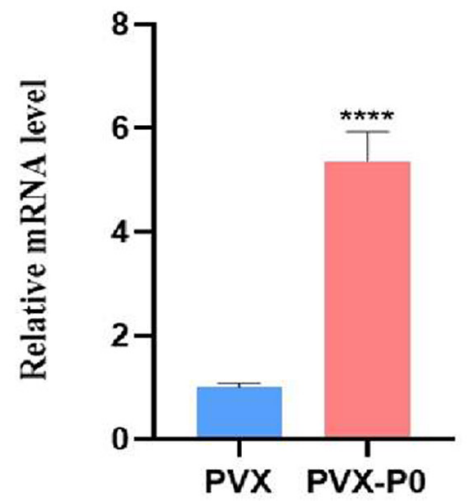

D

\section{H PVX-P0 PVX}

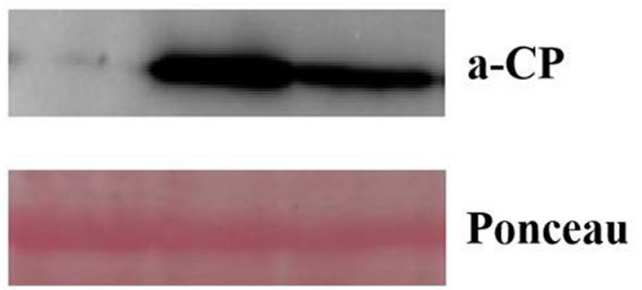

FIGURE 1 | PeVYV-derived PO protein influences potato virus X (PVX) pathogenesis. (A) Symptomatology of Nicotiana benthamiana crops infected with Agrobacterium cells carrying PVX alone or a recombinant PVX vector expressing ORF 0 of PeVYV. Images created at 5 days post-infiltration (dpi) and 10 dpi are shown. Three independent assays were conducted, and a minimum of four to six crops were utilized for each infiltration event. (B) $D A B$ staining for detecting $\mathrm{H}_{2} \mathrm{O}_{2}$ accumulation within PVX-PO- and PVX-infected N. benthamiana crops at 3 dpi. Results illustrated increased accumulations of $\mathrm{H}_{2} \mathrm{O}_{2}$ within PVX-PO-infected foliage. (C) RT-qPCR analysis of PVX CP RNA accumulation within inoculated leaves at 5 dpi. Results illustrate increased accumulations of PVX CP RNA in PVX-P0-infected foliage. Values are means \pm SDs. Highly significant differences ${ }^{* \star \star \star} \rho<0.0001$ ) between samples in each pair are indicated. (D) Western blotting analysis of PVX accumulation within infected $\mathrm{N}$. benthamiana plants at 5 to 7 dpi using a monoclonal antibody targeting PVX CP. Total protein was collected from upper non-infected foliage as indicated in (A). Two separate crops were used for extracting protein contents. H stands for total soluble proteins collected from N. benthamiana crop used for detecting PVX antibody specificity. Ponceau staining of Rubisco (large subunit) was used in loading control well.

wilting and necrotic lesions (Figure 1A). To validate whether the necrotic phenotype correlated with the $\mathrm{H}_{2} \mathrm{O}_{2}$ content, $\mathrm{DAB}$ reagent staining was used. As shown in Figure 1A, leaves inoculated with PVX-P0 induced $\mathrm{H}_{2} \mathrm{O}_{2}$ bursts, suggesting that PVX-P0 could act as a symptom inducer. RT-qPCR was used to investigate PVX RNA accumulation within the upper leaves. As shown in Figures 1B,C, crops infected with PVX-P0 displayed exacerbations in virulence, and these symptoms were closely correlated with upregulated expression of PVX RNAs. The quantification of RNA accumulation of PVX and the Western blotting analysis further indicated that $\mathrm{P} 0$ protein accumulation was significantly higher than that obtained with the PVX vector (Figure 1D). These results suggested that PeVYV P0 has major pathogenicity influences.

\section{F-Box Motif Is the Necrosis-Inducing Domain of PeVYV PO}

All inoculated plants were kept under constant conditions $\left(25^{\circ} \mathrm{C}\right.$ day $/ 22^{\circ} \mathrm{C}$ night, 16-h light $/ 8$-h dark $)$, and any symptoms were regularly recorded. Six plants were infected by each construct in a minimum of three independent experimental runs. At 5 days post-inoculation (dpi), mild mosaic symptoms were visible on the negative control PVX-infected plants. Similarly, $\mathrm{PVX}^{\mathrm{P}} 0^{\triangle}{ }^{57 a}$ caused more rapid, severe, visible necrotic symptoms in the infected foliage at $5 \mathrm{dpi}$, followed by necrotic lesions throughout the entire plant at $10 \mathrm{dpi}$ (Figure 2A), whereas PVX-

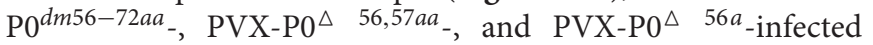
plants demonstrated only mild mosaic symptoms, which 


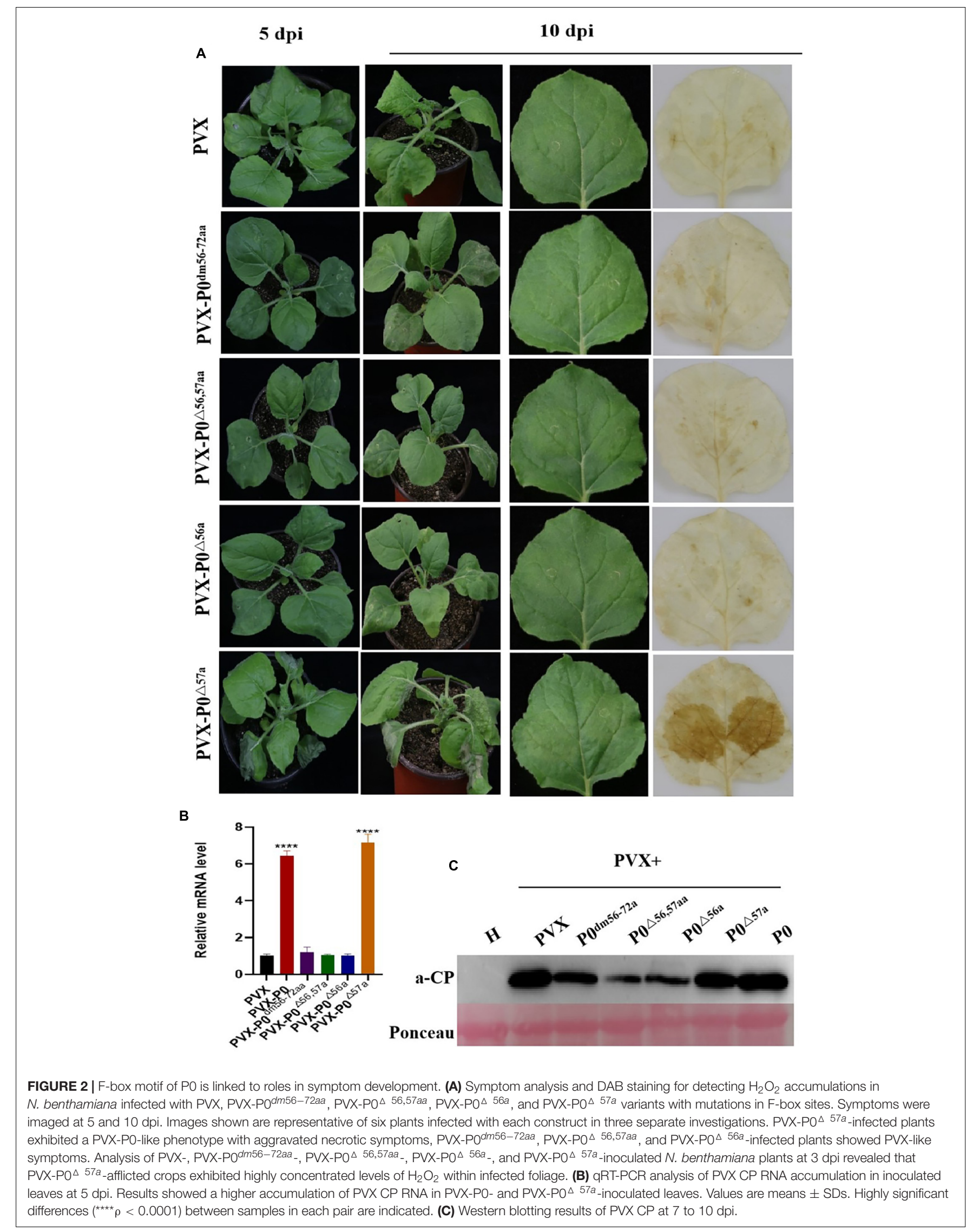


A 35S-GFP+

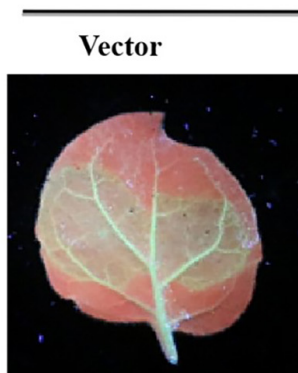

P0

P19
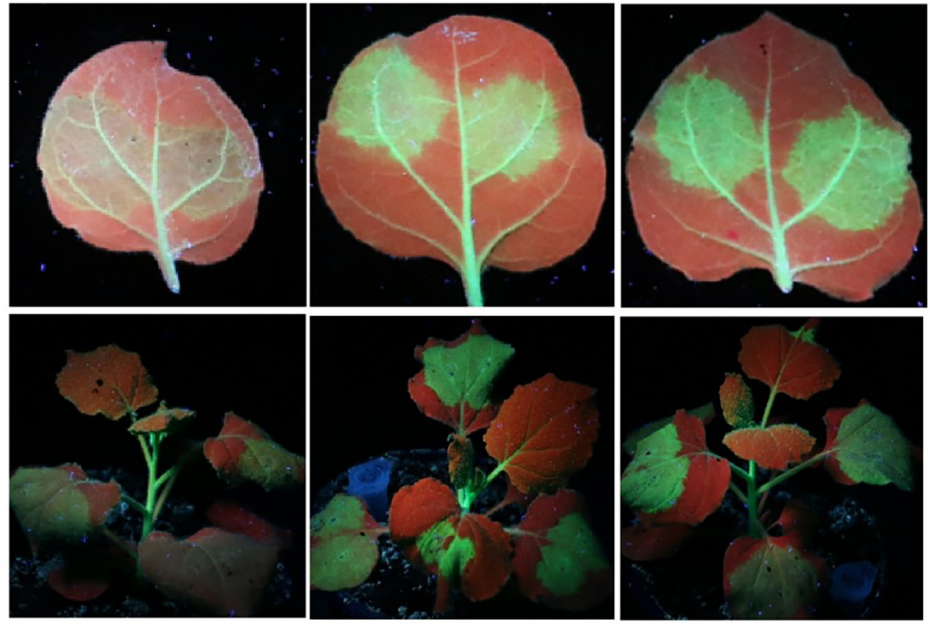

B

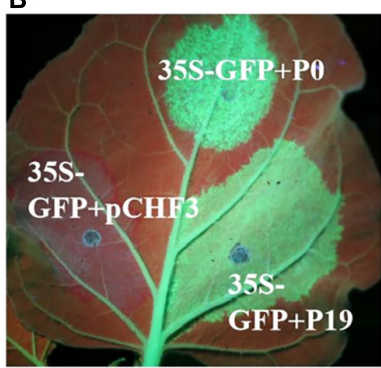

C

35S-GFP+

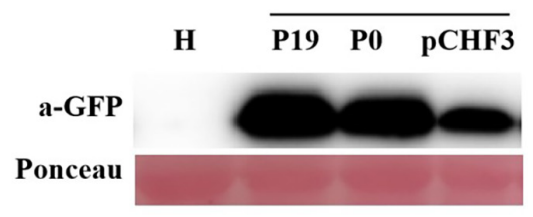

D

$16 \mathrm{C}$ 35S-GFP+

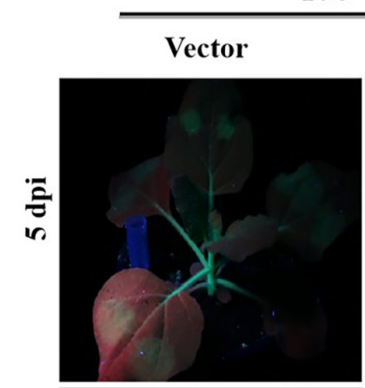

Po

P19
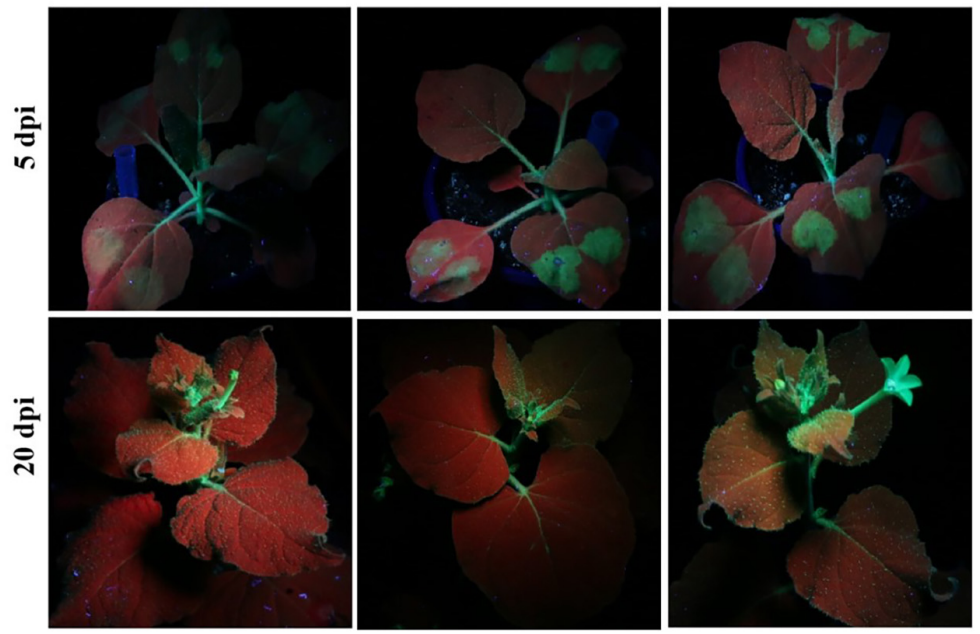

FIGURE 3 | Effect of PeVYV PO on GFP local/leaf silencing and systemic/whole plant silencing. (A) N. benthamiana 16c plants infected with Agrobacterium culture solutions carrying 35S-GFP and pCHF3 vectors expressing ORF 0 of PeVYV. Images were obtained under UV light with a yellow filter-mounted camera at 5 dpi. (B) Inhibition of local/leaf post-translational gene silencing (PTGS) in wild-type N. benthamiana crops. N. benthamiana crops were infected with Agrobacterium culture solutions as indicated. Images were collected under UV light at 5 dpi. (C) Western blotting analysis of GFP within infected N. benthamiana leaf patches utilizing a GFP monoclonal antibody. Total soluble protein content collected from N. benthamiana crops was needed for recognizing specificity of GFP antibody. Ponceau staining for Rubisco protein (large subunit) served as a loading control. (D) PeVYV PO NA silencing signal transduction within N. benthamiana 16c crops. Upper images illustrate N. benthamiana 16c crops infected with Agrobacterium cultures bearing 35S-GFP plus empty vector (systemic silencing), 35S-GFP plus PeVYV P0 (systemic silencing), or 35S-GFP plus P19 (no systemic silencing). Images collected using UV light at 20 dpi. 

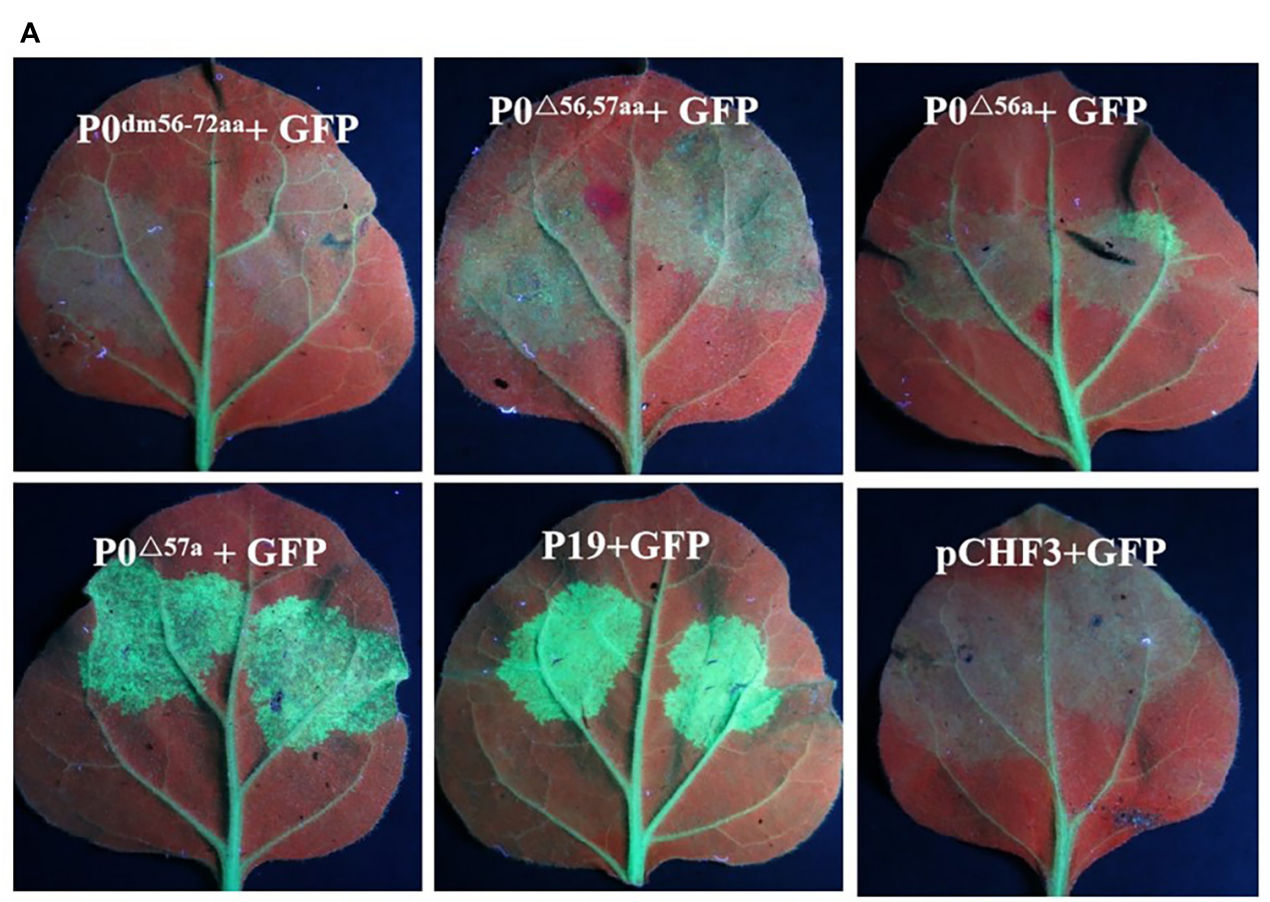

B

35S-GFP+

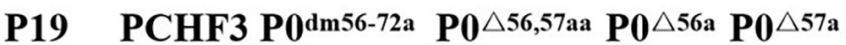

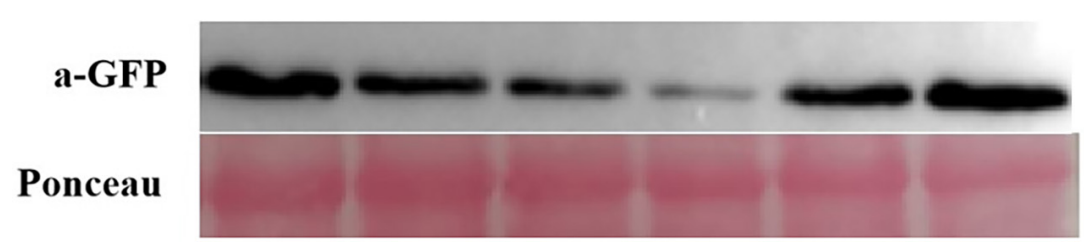

FIGURE 4 | Basic motif deletion in PeVYV PO affects RNA silencing capacity. (A) RSS activity within N. benthamiana plants. P0dm56-72aa, P0 $\triangle$ 56,57aa , P0 $\triangle$ 56a, and PO $\triangle 57 a$ were infiltrated with Agrobacterium cultures carrying specific constructs. Images were obtained under UV light at 5 days post-intervention (dpi). Three separate infiltration assays were performed, and four biological replicates (plants) were included in each assay. (B) Western blotting analyses of GFP expression within infiltrated foliage utilizing GFP-specific monoclonal antibodies. Ponceau staining for Rubisco (large subunit) served as a loading control.

was not dissimilar to the findings obtained with plants infected with PVX alone.

Figure 2A also revealed that the $\mathrm{H}_{2} \mathrm{O}_{2}$ content was exacerbated within systemic $\mathrm{PVX}^{-} \mathrm{P0}^{\triangle} 57 a_{\text {-infected leaves at }}$ 10 dpi. Conversely, no visible $\mathrm{H}_{2} \mathrm{O}_{2}$ content was identified within PVX-P0 ${ }^{d m 56-72 a a}, \mathrm{PVX}_{-} \mathrm{P}^{\triangle} 56,57 a a$, and $\mathrm{PVX}-\mathrm{P} 0^{\triangle} 56 a_{-}$ inoculated foliage.

Fluorescence-based RT-qPCR methodologies were used to quantify PVX RNA within the upper leaves at $5 \mathrm{dpi}$. As illustrated in Figure 2B, exacerbated virulence was closely correlated with upregulated expression of the PVX transcript. PVX-P0- or PVX$\mathrm{PO}^{\triangle 57 a}$-infected plants exhibited upregulated expression of viral RNA compared with plants infected with PVX-P0dm56-72aa, $\mathrm{PVX}^{\mathrm{P} 0} 0^{\triangle 56,57 a a}$, or PVX-P0 ${ }^{\triangle} 56 a$ within the upper leaf foliage.

Western blotting techniques were also adopted to quantify the viral RNA levels, and the results highlighted that PVX, PVX$\mathrm{P} 0$, and $\mathrm{PVX}-\mathrm{P} 0^{\triangle} 57 a$ attained increased viral RNA accumulation within the inoculated leaves at $5 \mathrm{dpi}$ (Figure 2C). In essence, such data and consequent results provide evidence showing that AA 57 of the PeVYV-P0 protein leads to exacerbated symptom development and together aggravates the proliferative rate of PVX to higher levels.

\section{PeVYV PO Inhibits Local (Though Not Systemic) Gene Silencing at the Transcriptional Level}

Recent findings demonstrate that P0 proteins of multiple Polerovirus species are effective RSSs of PTGS through either local or systemic inhibition of such RNA regulatory processes. To confirm whether P0 can truly inhibit PTGS, concomitant infiltration assays were developed for GFPtransgenic $N$. benthamiana 16c plants, as described earlier. At 5 dpi, P0 + GFP-expressing leaves demonstrated 

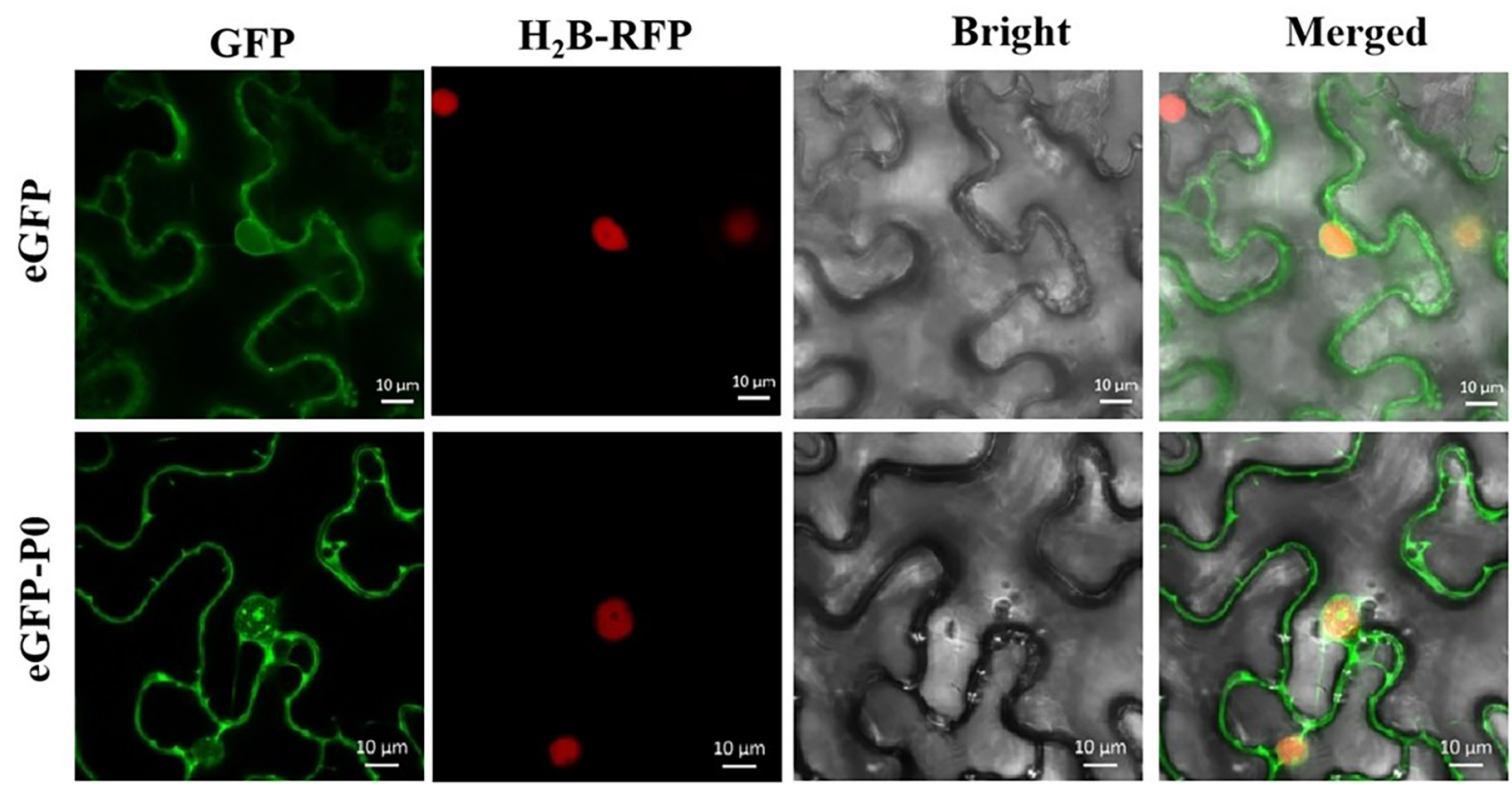

FIGURE 5 | Subcellular localization of PeVY PO within Nicotiana benthamiana foliage. All pCHF3-eGFP-derived GFP expression-linked fluorescence was dissipated uniformly within cytoplasm/nucleus. P0-GFP fusion protein derived from pCHF3-eGFP is localized within nucleus, particularly within nucleolar regions of virus-infiltrated leaves of $N$. benthamiana. All images were visualized with a confocal microscope (Zeiss LSM880) at 36- to 48-h post-infiltration. Independent infiltration experiments were conducted three times on three separate occasions, and five to six cells were assessed during each investigation.

elevated, visible green fluorescence under UV light, which was closely similar to the fluorescent effects exhibited by P19 + GFP-expressing leaves (Figure 3A). This finding was closely correlated with the upregulated expression of GFP, as revealed by Western blotting analyses. However, 35S$\mathrm{GFP}+\mathrm{P} 0 / 35 \mathrm{~S}$-GFP + P19-inoculated foliage exhibited more intense GFP-based fluorescence (Figure 3B). The results from Western blotting analyses were also closely correlated with the GFP fluorescence findings (Figure 3C). In addition, infected crops were scrutinized for possible systemic/wholeplant silencing activities in the upper foliage (i.e., young) at 20 dpi (Figure 3D). The majority of veins within the youngest (uppermost) leaves of the $35 \mathrm{~S}$-GFP + P0-infected plants exhibited red coloration under UV illumination, suggesting the onset of RNA silencing mechanisms. Conversely, the GFP fluorescence levels were maintained within the majority of $16 \mathrm{c}$ plant foliage leaves inoculated with 35S-GFP + P19. Taken together, the results verify that PeVYV P0 is a powerful local gene silencing suppressor, although it was unable to achieve systemic silencing.

\section{F-Box Motif Is Needed for PeVYV P0-Induced Post-translational Gene Silencing Inhibition}

Previous studies have suggested that multiple VSR F-boxes are pivotal for RSS activities, and these findings spark the need to confirm such findings, as described earlier. UV-lightmonitored leaves demonstrated that the coexpression of 35SGFP with empty vector, 35 S-GFP and $\mathrm{P}^{d m 56-72 a a}, \mathrm{P}^{\triangle 5}$ 56,57aa, or $\mathrm{P}^{\triangle} 56 a$ led to a lack of GFP-based fluorescence at $5 \mathrm{dpi}$, whereas 35 S-GFP + P19 or 35 S-GFP $+\mathrm{P}^{\triangle} 57 a$ resulted in intense green fluorescence (Figure 4A). This finding was corroborated through analyses of the relative GFP levels in corresponding leaf patches (Figure 4B), which indicated that the 56LPFLLSSQCPFLSGNTP72 motif and AA 56 of PeVYV are needed by PeVYV P0 to successfully inhibit RNA silencing. These results suggested that AA 56 of the F-box motif is essential for P0 protein function in RNA silencing suppression.

\section{F-Box Motif Changes the Subcellular Localization of PeVYV PO}

The development of tools for analyzing subcellular localization and thereby tracking virus-derived proteins was a major step toward unraveling potential roles throughout the course of viral infection. Figure 5 highlights the results from the assays of subcellular localizations within the plants conducted in this study. As predicted, free GFP was identified within the cell membrane and nucleus in a quasiuniform distribution. The P0-eGFP fusion was found to accumulate within the cell membrane and nucleus, particularly within the nucleolus.

Figure 6 shows that the $\mathrm{P}^{\triangle} 57 a_{\text {-eGFP fusion aggregated }}$ intensely within the nucleus, particularly within the nucleolus. $\mathrm{P}^{d m 56-72 a a}$-eGFP, $\mathrm{P}^{\triangle}{ }^{56,57 a a_{-}}$-eGFP, and $\mathrm{P}^{\triangle}{ }^{56 a}$-eGFP were found to accumulate in the cell membrane and nucleus, without any nucleolar presence. In essence, the results indicated that the 56LPFLLSSQCPFLSGNTP72 motif and AA 56 of PeVYV P0 are essential for P0 subnuclear localization. 


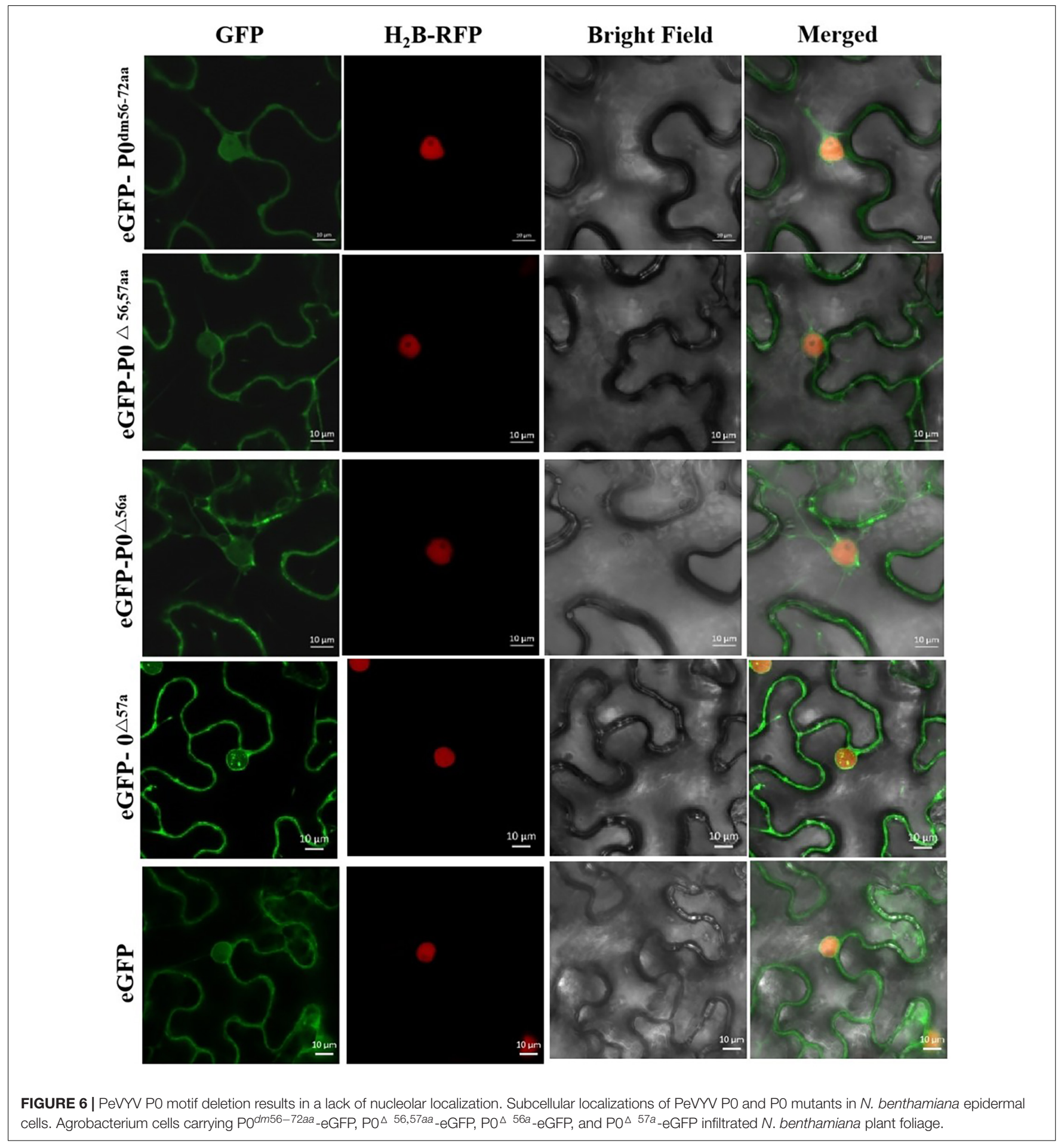

\section{DISCUSSION}

Polerovirus-derived P0 proteins are highly effective RSSs in a wide spectrum of viruses, including TuYV, cucurbit aphidborne yellows virus (CABYV), potato leaf roll virus (PLRV), cereal yellow dwarf virus (CYDV), beet western yellows virus (BWYV), cucurbit aphid-borne yellows virus (CABYV), cotton leaf roll dwarf virus (CLRDV), melon aphid-borne yellow virus (MABYV), beet mild yellowing virus (BMYV), maize yellow dwarf virus-RMV2 (MYDV-RMV2), and brassica yellows virus (BrYV) (Pazhouhandeh et al., 2006; Han et al., 2010; Almasi et al., 2015; Cascardo et al., 2015; Chen et al., 2016; Wang et al., 2018; Li et al., 2019). Concomitant-acting roles are typically presented by RSSs, and these include the fulfillment 
of other non-silencing suppression functions throughout the infective course. Consequently, VSRs have the ability to modulate heterologous system pathogenicity in differing manners (Sharma et al., 2010). TuYV P0 ${ }^{T u}$, potato leafroll virus $\mathrm{P} 0^{P L}$, and CABYV $\mathrm{P} 0{ }^{C A}$ induce hypersensitive responses in N. glutinosa accession TW59 (Wang et al., 2015). Conforming the findings from previous investigations, PVX-derived PeVYV P0 expression induced leaf curl manifestations and necrotic presentations that were completely unique compared with the typical symptoms observed in response to PVX infections, whereas mutagenesis of the F-box motif and AA 57, which are both found within the P0 protein, did not lead to necrosis within the afflicted plants. This finding indicated that the F-box-like domain of PeVYV P0 is key in such pathogenic processes, whereas AA 57 plays similar roles in pathogenesis (albeit to a lower extent) and in conferring virulence properties.

The F-box-like domain (N-terminal) of $\mathrm{P} 0$ has been identified to play pivotal roles in RSS activities (Han et al., 2010; Fusaro et al., 2012). There are two conserved domains in the P0 protein: the consensus F-box-like motif (LPXX(L/I)X10$13 \mathrm{P}$ ) and the Phe/Trp (FW) remnants at the C-terminal consensus sequence [(K/R) IYGEDGX3FWR] (Han et al., 2010; Fusaro et al., 2012; Almasi et al., 2015; Chen et al., 2016). MYDV-RMV2 P0 functions such as a typical F-boxlike motif, and mutagenesis of Ala [positions 67, 68, and 81 in the F-box-like motif (67LPxx81P)] fully inhibits P0-induced RSS activities (Wang et al., 2018). The F-box-like domain is essential for SSP functional roles, although the LP motif is redundant for such activities. The introduction of a $3 \times \mathrm{AA}$ substitution event within pea enation mosaic virus-1 (PEMV1) P0 (leucine-124, proline-125, and proline-133 converted to alanine residues) produced a protein (P0PE $\triangle \mathrm{LPP}$ ) without RSS activities, which is actually the opposite of our previous findings (Fusaro et al., 2012).

Trp 212 is a key player in the RSS mechanism within the MABYV P0 protein (Han et al., 2010). Our findings are similar to those found for the P0 protein of Polerovirus by previous authors. Our results show that the F-box-like domain and AA 56 of P0 are essential for green fluorescence in $N$. benthamiana 16c. In addition, PeVYV P0 inhibits local, although not systemic, RNA silencing mechanisms. This finding is similar to those found with TuYV, CABYV, PLRV (EU variant), MABYV, and BMYV P0 proteins, all of which are SSPs only at the local RNA silencing level (Han et al., 2010). However, the P0 protein found in other viruses, such as sugarcane yellow leaf virus (SCYLV) and PEMV-1 (genus Enamovirus), manages to perform RSS functions at the local and systemic levels (Li et al., 2019), which highlights that the P0 proteins from multiple viruses derived from Poleroviruses display different RSS capacities.

The nature of the subcellular localizations leads to a better understanding of viral interactions with key host molecular players and the viral roles during the course of infection. Similar to poleroviral P0 proteins, PEMV-1 P0 is located in the nucleus (Fusaro et al., 2012). MYDV-RMV2 P0 has been found in both the nucleus and cytoplasm (Wang et al., 2018). This study revealed that the PeVYV
P0 protein tended to exhibit a complex localization, with an intense presence within the cytoplasm, nucleus, and nucleolus. The mutagenesis of AA 56 on P0 led to the dissipation of the protein from the cytoplasm/nucleus, indicating that the mutated variant of this protein was localized in the nucleolus.

Point mutations within the P0 F-box motif could inhibit SKP1 interactions, consequently lowering AGO1 protein destabilizing events and eventually leading to viral pathogenicity (Derrien et al., 2012, 2018; Li et al., 2019). The TuYV, CABYV, and BrYV P0 proteins contain an F-box-like domain that interacts with SKP1 (Li et al., 2019). BrYV-A P0 BrA also interacts with NbRAF2, which consequently alters the localization distribution profile to enhance viral invasion (Sun et al., 2018). However, P0 fails to help proteasome-directed degradation events, as predicted, and has rather been found to recognize AGO1-derived DUF1785 domain degradation and consequently initiate autophagy-mediated AGO1 (Derrien et al., 2012, 2018). Most likely, the P0 protein encoded by PeVYV interacts with related genes in the ubiquitination or AGO pathways or other verified host factors, and the results scientifically uncover the novel molecular mechanisms underlying PeVYVhost interactions.

\section{CONCLUSION}

Our results validated that the $\mathrm{P} 0$ protein encoded by the initial ORF of PeVYV P0 has RSS activity, induces hypersensitivity responses that regulate viral propagation, and leads to apoptosis. All these activities tend to stem from AA 56 or AA 57 of the P0-derived F-box-like domain. The outcomes obtained here suggest that further studies should be conducted on the molecular mechanisms of amino acids of the F-box domain of $\mathrm{P} 0$ protein in the interaction of PeVYV with plants.

\section{DATA AVAILABILITY STATEMENT}

The original contributions presented in the study are included in the article/supplementary material, further inquiries can be directed to the corresponding author.

\section{AUTHOR CONTRIBUTIONS}

LW, PT, and CL carried the experimental work. SZ and XHY collected and analyzed the data. XLY, XZ, and YL designed study, guided data interpretation, and wrote the manuscript. All authors approved the manuscript before it was submitted by the corresponding author.

\section{FUNDING}

This research was granted by the National Natural Science Foundation of China (32060606) and the China Agriculture Research System (CARS-23-D-02). 


\section{REFERENCES}

Afouda, L., Kone, D., Zinsou, V., Dossou, L., Kenyon, L., Winter, S., et al. (2017). Virus surveys of Capsicum spp. in the Republic of Benin reveal the prevalence of pepper vein yellows virus and the identification of a previously uncharacterised polerovirus species. Arch. Virol. 162, 1599-1607. doi: 10.1007/s00705-0173274-8

Agrofoglio, Y. C., Delfosse, V. C., Casse, M. F., Hopp, H. E., Bonacic Kresic, I., Zieglergraff, V., et al. (2019). P0 protein of Cotton leafroll dwarf virus-atypical isolate is a weak RNA silencing suppressor and the avirulence determinant that breaks the cotton Cbd gene-based resistance. Plant Pathol. 68, 1059-1071. doi: $10.1111 /$ ppa.13031

Alabi, O. J., Rwahnih, M. A., Jifon, J. L., Gregg, L., Crosby, K., and Mirkov, T. E. (2015). First report of Pepper vein yellows virus infecting pepper (Capsicum spp.) in the United States. Plant Dis. 99:1656. doi: 10.1094/pdis-03-15-0329-pdn

Alfaro, F. A., ElShafie, E. E., Ali, M. A., El Bashir, O. O. A., Córdoba-Sellés, M. C., and Font San Ambrosio, M. I. (2014). First report of Pepper vein yellows virus infecting hot pepper in Sudan. Plant Dis. 98:446. doi: 10.1094/pdis-03-14-0251pdn

Ali, M., Anwar, S., Shuja, M. N., Tripathi, R. K., and Singh, J. (2018). The genus Luteovirus from infection to disease. Eur. J. Plant Pathol. 151, 841-860. doi: 10.1007/s10658-018-1425-8

Almasi, R., Miller, W. A., and Ziegler-Graff, V. (2015). Mild and severe cereal yellow dwarf viruses differ in silencing suppressor efficiency of the P0 protein. Virus Res. 208, 199-206. doi: 10.1016/j.virusres.2015.06.020

Avelar, S., Ramossobrinho, R., Conner, K., Nichols, R. L., Lawrence, K. S., and Brown, J. K. (2020). Characterization of the complete genome and P0 protein for a previously unreported genotype of cotton leafroll dwarf virus, an introduced Polerovirus in the United States. Plant Dis. 104, 780-786. doi: 10.1094/pdis-06-19-1316-re

Balzergue, C., Dartevelle, T., Godon, C., Laugier, E., Meisrimler, C., Teulon, J. M., et al. (2017). Low phosphate activates STOP1-ALMT1 to rapidly inhibit root cell elongation. Nat. Commun. 8:15300.

Bryksin, A. V., and Matsumura, I. (2018). Overlap extension PCR cloning: a simple and reliable way to create recombinant plasmids. Biotechniqes 48, 463-465. doi: $10.2144 / 000113418$

Cascardo, R. S., Arantes, I. L. G., Silva, T. F., Sachetto-Martins, G., Vaslin, M. F. S., and Correa, R. L. (2015). Function and diversity of P0 proteins among Cotton leaf roll dwarf virus isolates. Virol. J. 12:123. doi: 10.1186/s12985-015-0356-7

Chen, S., Jiang, G., Wu, J., Liu, Y., Qian, Y., and Zhou, X. (2016). Characterization of a novel polerovirus infecting maize in China. Viruses 8:120. doi: 10.3390/ v8050120

Derrien, B., Baumberger, N., Schepetilnikov, M., Viotti, C., De Cillia, J., ZieglerGraff, V., et al. (2012). Degradation of the antiviral component ARGONAUTE1 by the autophagy pathway. Proc. Natl. Acad. Sci. U.S.A. 109, 15942-15946. doi: 10.1073/pnas.1209487109

Derrien, B., Clavel, M., Baumberger, N., Iki, T., Sarazin, A., Hacquard, T., et al. (2018). A suppressor screen for AGO1 degradation by the viral F-Box P0 protein uncovers a role for AGO DUF1785 in sRNA duplex unwinding. Plant Cell 30, 1353-1374. doi: 10.1105/tpc.18.00111

Dombrovsky, A., Glanz, E., Pearlsman, M., and Antignus, Y. (2010). Characterization of Pepper yellow leaf curl virus, a tentative new Polerovirus species causing a yellowing disease of pepper. Phytoparasitica 38, 477-486. doi: $10.1007 / \mathrm{s} 12600-010-0120-\mathrm{x}$

Fusaro, A. F., Correa, R. L., Nakasugi, K., Jackson, C., Kawchuk, L., Vaslin, M. F., et al. (2012). The Enamovirus P0 protein is a silencing suppressor which inhibits local and systemic RNA silencing through AGO1 degradation. Virology 426, 178-187. doi: 10.1016/j.virol.2012.01.026

Han, Y. H., Xiang, H. Y., Wang, Q., Li, Y. Y., Wu, W. Q., Han, C. G., et al. (2010). Ring structure amino acids affect the suppressor activity of melon aphidborne yellows virus $\mathrm{P} 0$ protein. Virology 406, 21-27. doi: 10.1016/j.virol.2010.06. 045

Knierim, D., Tsai, W. S., and Kenyon, L. (2013). Analysis of sequences from field samples reveals the presence of the recently described pepper vein yellows virus (genus Polerovirus) in six additional countries. Arch. Virol. 158, 1337-1341. doi: 10.1007/s00705-012-1598-y

Li, Y. Y., Sun, Q., Zhao, T. Y., Xiang, H. Y., Zhang, X. Y., Wu, Z. Y., et al. (2019). Interaction between Brassica yellows virus silencing suppressor P0 and plant SKP1 facilitates stability of P0 in vivo against degradation by proteasome and autophagy pathways. New Phytol. 222, 1458-1473. doi: 10.1111/nph.15702

Liu, M. Y., Liu, X. N., Li, X., Zhang, D. Y., Dai, L. Y., and Tang, Q. J. (2016). Complete genome sequence of a Chinese isolate of Pepper vein yellows virus and evolutionary analysis based on the CP, MP and RdRp coding regions. Arch. Virol. 161, 677-683. doi: 10.1007/s00705-015-2691-9

Murakami, R., Nakashima, N., Hinomoto, N., Kawano, S., and Toyosato, T. (2011). The genome sequence of pepper vein yellows virus (family Luteoviridae, genus Polerovirus). Arch. Virol. 156, 921-923. doi: 10.1007/s00705-011-0956-5

Pazhouhandeh, M., Dieterle, M., Marracco, K., Lechner, E., Berry, B., Brault, V., et al. (2006). F-box-like domain in the polerovirus protein P0 is required for silencing suppressor function. Proc. Natl. Acad. Sci. U.S.A. 103, 1994-1999. doi: 10.1073/pnas.0510784103

Sharma, P., Ikegami, M., Jaeger, K. E., Graf, A., Wigge, P. A., and Saitou, N. (2010). Tomato leaf curl Java virus V2 protein is a determinant of virulence, hypersensitive response and suppression of post transcriptional gene silencing. Virology 396, 85-93. doi: 10.1016/j.virol.2009.10.012

Sun, Q., Li, Y. Y., Wang, Y., Zhao, H. H., Zhao, T. Y., Zhang, Z. Y., et al. (2018). BrYV P0 protein impairs the antiviral activity of NbRAF2 in Nicotiana Benthamiana. J. Exp. Bot. 69, 3127-3139. doi: 10.1093/jxb/ery131

Tan, W. P., Dong, Y. Z., Sun, X. H., Liang, Y. C., Liu, H. X., and Zhu, X. P. (2015). The first identification of Pepper vein yellows virus in Shandong Province, China. Plant Dis. 99:1288. doi: 10.1094/pdis-12-14-1333-pdn

Villanueva, F., Castillo, P., Font, M. I, Alfaro-Fernández, A., Moriones, E., and Navas Castillo, J. (2013). First report of Pepper vein yellows virus infecting sweet pepper in Spain. Plant Dis. 97:1261. doi: 10.1094/pdis-04-13-0369-pdn

Wang, F., Zhao, X., Dong, Q., Zhou, B. G., and Gao, Z. L. (2018). Characterization of an RNA silencing suppressor encoded by Maize yellow dwarf virus-RMV2. Virus Genes 54, 570-577. doi: 10.1007/s11262-018-1565-0

Wang, K. D., Empleo, R., Nguyen, T. T. V., Moffett, P., and Sacco, M. A. (2015). Elicitation of hypersensitive responses in Nicotiana glutinosa by the suppressor of RNA silencing protein P0 from poleroviruses. Mol. Plant Pathol. 16, 435-448. doi: 10.1111/mpp.12201

Wang, L. S., He, Q. C., Chen, X. J., He, H. Y., Yang, X. H., Lu, Q. H., et al. (2017). First report of Pepper vein yellows virus infecting tobacco (Nicotiana tabacum L.) naturally in China. Plant Dis. 101:1556. doi: 10.1094/pdis-01-17-0071-pdn Yonaha, T., Toyosato, T., Kawano, S., and Osaki, T. (1995). Pepper vein yellows virus, a novel luteovirus from bell pepper plants in Japan. Jpn. J. Physiol. 61, 178-184. doi: 10.3186/jphytopath.61.178

Zhang, S. B., Zhao, Z. B., Zhang, D. Y., Liu, Y., Luo, X. W., Liu, J., et al. (2015). First report of pepper vein yellows virus infecting red pepper in mainland China. Plant Dis. 99:1190. doi: 10.1094/pdis-01-15-0025-pdn

Conflict of Interest: The authors declare that the research was conducted in the absence of any commercial or financial relationships that could be construed as a potential conflict of interest.

Publisher's Note: All claims expressed in this article are solely those of the authors and do not necessarily represent those of their affiliated organizations, or those of the publisher, the editors and the reviewers. Any product that may be evaluated in this article, or claim that may be made by its manufacturer, is not guaranteed or endorsed by the publisher.

Copyright (®) 2021 Wang, Tian, Yang, Zhou, Zhang, Li, Yang and Liu. This is an open-access article distributed under the terms of the Creative Commons Attribution License (CC BY). The use, distribution or reproduction in other forums is permitted, provided the original author(s) and the copyright owner(s) are credited and that the original publication in this journal is cited, in accordance with accepted academic practice. No use, distribution or reproduction is permitted which does not comply with these terms. 